\title{
Exploring Product Fit And Brand Fit In Brand Alliances: Establishing The Role Of Categorization Processes
}

\author{
Andrew T. Norman, Drake University, USA
}

\begin{abstract}
The marketing strategy of brand alliance has received considerable attention in marketing literature. A significant factor in the study of how consumers process brand alliances is the concept of "fit". Specifically, it has been shown that consumers evaluate the extent to which the brands and products in a brand alliance are congruent.

While the concept of fit has been addressed in brand alliance research, little is known about what fit really is. This paper seeks to establish the processes by which consumers make evaluations of both product and brand fit. Based on a review of the relevant literature, it is proposed that consumers use higher-order categorization processes in evaluating brand alliances.

A $2 \times 2$ experiment was conducted to test multiple hypotheses. Results confirm these hypotheses and confirm that consumers do indeed conduct a higher-order category search as they evaluate brand alliances. Based on this work, an entire field of research can now be applied to the study of this promotional strategy. While various studies in the recent past have examined factors affecting the processing of brand alliances, there remains a great deal to be learned about how brand alliance information is processed and what factors affect both cognitive and evaluative outcomes. Applications of categorization theories open new avenues in the study of this area of consumer research.
\end{abstract}

Keywords: Brand Alliances; Co-Branding; Brand Management, Categorization

\section{INTRODUCTION}

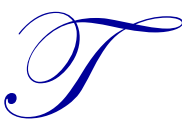

he practice of cooperative brand activities by marketers is as prevalent as ever. Mastercard reports that co-branded credit cards account for approximately 50 percent of all credit card spending and that co-branded card spend is growing at a faster rate than that of non co-branded cards (Mastercard site). It's that kind of promise that has the likes of American Airlines, Paypal, Carnival Cruise Lines, Overstock.com, and hundreds of other companies teaming up with credit card providers.

Cooperative brand activities are not confined to co-branded credit cards. Other recent examples include Betty Crocker Hershey's Ultimate Fudge Supreme Brownie Mix, the Fiat 500 Barbie (a limited edition commemorating Barbie's $50^{\text {th }}$ birthday), T.G.I. Friday's Jack Daniel's menu selections, and a host of Proctor \& Gamble combos like Crest plus Scope and Tide with Febreze. The objectives marketers outline for pursuing such practices vary as much as the outcomes.

In practice as well as in academic research, cooperative brand activities have gone by various terms, including brand alliances, co-branding, co-marketing, cross-promotion, joint branding, and joint sales promotion. While subtle characteristics distinguish these promotional tactics from one another, this research focuses specifically on cooperative brand promotions characterized by the definition of brand alliances. Brand alliances involve the association or combination of two or more individual brands and products in a single context and are commonly represented through some means of promotion (Rao and Ruekert, 1994; Simonin and Ruth, 1998). Key empirical studies have contributed to the knowledge of how consumers process such alliances. Specifically, it has been shown that brand alliances have an impact on the attitude toward each component brand (Simonin and Ruth, 1998), exhibit 
effects on brand awareness, accessibility, beliefs, and attitudes for newly introduced brands (Samu et al., 1999), affect shopping and purchase intentions (Arnett et al., 2010), and create a link whereby attribute and quality information transfers from one brand to another (Levin and Levin, 2000; James 2005; Rao et al., 1999).

In the search to establish the processes by which consumers evaluate the combination of multiple brands in a single setting, most of these studies address the issue of "fit" between the entities involved in a brand alliance. While the concept of brand alliance fit has most often been operationalized very simply as product complementarity (Bendik, 2015), others establish and define two separate dimensions of brand alliance fit (Hao, 2015). Simonin and Ruth (1998) provide strong evidence that as consumers process a brand alliance, they simultaneously evaluate the extent to which the product categories involved in a brand alliance are related or compatible (product fit) as well as the degree to which the images of both brands are consistent with or complementary to each other (brand fit).

While multiple dimensions of fit have been recognized as key variables affecting consumer perceptions of brand alliances, a conceptual understanding of the psychological processes involved in making fit evaluations is lacking. In spite of the extent to which fit issues have been addressed in the study of brand alliances, we know very little with respect to what "fit" really is. A theoretical knowledge of fit would serve to open new directions in the understanding of how consumers perceive, evaluate, and process this type of marketing strategy.

The purpose of this paper is primarily to establish the process that consumers follow when making evaluations of brand alliance fit. As a secondary purpose, this paper will provide confirming evidence as to the existence of two distinct dimensions of brand alliance fit, product fit and brand fit. To accomplish these purposes, this paper looks at the vast literature on categorization as well as the application of categorization processes in the marketing strategies of brand extension and brand alliance. Based on this review of the literature, hypotheses regarding the nature of both product fit and brand fit are established. The methods and results of an experimental study that support these hypotheses are then discussed.

\section{A THEORETICAL FOUNDATION FOR ASSESSING FIT}

In an area of marketing research relevant to the study of brand alliances, categorization theory has been applied as a basis for understanding the extent to which a brand extension fits with the core brand (Aaker and Keller, 1990; Bhat and Reddy, 2001; Boush and Loken, 1991; Broniarczyk and Alba, 1994; Kreuzbauer and Malter, 2005; Park et al., 1991). A category has been defined as "two or more distinguishable objects that are treated equivalently" (Mervis and Rosch, 1981). In the sense that products with the same brand label are viewed as distinguishable objects that are treated equivalently, a brand satisfies this definition of a category.

Studies that have examined the process by which a brand extension is considered a member of the brand category commonly recognize the feature matching approach to categorization (Mervis and Rosch, 1981; Rosch, 1978; Smith et al., 1974; Smith and Medin, 1981). This approach asserts that as consumers consider a brand extension, the individual features or attributes of the extension are considered relative to the individual attributes of the core brand. The greater the number of attributes the extension has in common with the brand, the more the extension meets the criteria for membership in the brand category. The identification of this attribute-based approach to brand membership is significant because it has been shown that brand-specific associations transfer to a brand extension to the extent that the extension is considered a member of the brand category (Boush and Loken, 1991). Thus, perceived fit between a brand extension and a brand category moderates this transfer of associations.

A brand alliance is similar to a brand extension in that both involve some type of simultaneous evaluation of two different products. Additionally, some type of attitude transfer is likely to take place between the two products involved in either strategy.

However, a brand extension is always evaluated against an existing brand that serves as a benchmark or reference point. This readily allows for the comparing of attributes. In a brand alliance, there are exist two or more distinctive brands and, most commonly, neither serve as an identifiable benchmark. Additionally, a brand extension very commonly incorporates a product category that is logically related to the products that are established members of 
the brand family. Brand alliances, on the other hand, often involve two entirely different product categories that may initially seem to have little similarity when comparing features alone.

Unlike brand extensions, which involve only one brand, brand alliances involve more than one brand as well as more than one product type. Thus, the feature matching approach to fit for cooperative brand activities is inadequate as it is possible that the two products involved will share few feature similarities (i.e., American Airlines and VISA share few if any similar features).

However, basic principles of similarity and categorization are applicable to an understanding of brand alliance fit. This will first be demonstrated by examining the dimension of product fit. Product fit is defined as the extent to which the product categories incorporated in the brand alliance are consistent or complementary with one another (Simonin and Ruth, 1998). It is proposed here that the process involved in making an evaluation of fit between two product categories in a brand alliance is based on a hierarchical or higher-order categorization process.

Hierarchical categorization is defined by a few key concepts. A category is basically defined in the same manner identified previously - a number of objects that are considered equivalent based on some set of criteria (Mervis and Rosch, 1981). Higher-order categorization identifies that a taxonomy of categories can be conceived as varying along a vertical dimension based on different levels of inclusiveness. Thus, the terms "collie", "dog", "mammal", "animal", and "living thing" each represent a category. Each of these categories is at a different level of inclusiveness or abstraction, with "collie" representing the lowest, most concrete level and "living thing" representing the highest, most abstract level. Each category includes all categories at lower levels of abstraction. Thus, "living thing" encompasses all categories listed while "collie" doesn't include any categories other than itself.

Two products joined by a brand alliance may not seem to share any similarity of attributes on the basis of comparing the lower order (concrete) categories to which they belong. However, when a higher-order (abstract) category to which both categories can be considered members is considered, comparable attributes may be found. Consider the example of a brand alliance involving the jointly branded credit card American Airlines Visa in which all purchases made on the Visa card accumulate frequent flier miles to be used on American Airlines flights. If the attributes of each product are considered at the concrete category level of "airline service" and "credit card service", it is unlikely that common attributes will emerge. However, consider the higher-order category of "services useful for travel", which seems to readily come to mind in consideration of airline service and credit card service. Both products now seem to share very key common attributes and can be considered members of the same higher-order category. It should be noted that the selection of the higher-order category in this case is hardly random. It is assumed that the evaluation of two lower order categories that share no common features at a concrete level would elicit a search for a higher-order category that would provide the best fit.

This does not imply that a search will continue until a match is found. Some searches are likely to be more difficult than others. The higher a person has to search in the taxonomy of categories to find an appropriate higher-order category that will bind the two products, the more abstract that higher-order category becomes. Additionally, the higher the search climbs on a taxonomy of categories, the more elaboration is required for the task. If a search for a common category becomes too difficult, an individual may "give up", and merely conclude that there is no fit between the two objects. The level of abstraction of the binding higher-order category is, thus, a significant factor in assessing fit strength.

While a higher level of abstraction corresponds to greater inclusiveness, it also corresponds to fewer similar attributes amongst the members of such a category. Thus, members of the "credit card service" category share a greater number of similar attributes than the members of the "services useful for travel" category. This creates additional implications for brand alliances. Because abstract categories are broader, an evaluation of product fit based on such categories should be weaker than an evaluation of fit based on more concrete, lower order categories. This implies that the fewer levels that exist between each product category and a higher-order category to which they both belong, the stronger the level of fit.

To illustrate, if a brand alliance were to involve a basketball and basketball shoes as two product types, the common higher-order category that would likely emerge in the mind of a consumer is "items used to play basketball." Notice 
that the dynamics of this combination allow the shoes and the ball to be evaluated based on a relatively concrete higher-order category. If, however, the promotion involved a soccer ball and basketball shoes, the emerging higherorder category might be "sports equipment", a broader and more abstract category. As the level of fit between two products gets weaker, any higher-order category that could be used to group the two would become more abstract.

Although the dimension of brand fit is distinct from product fit, principles of categorization similar to those just used to describe product fit can be applied. Brand fit is defined as the degree to which the brand images of both brands are consistent with or complementary to each other (Simonin and Ruth, 1998). When a consumer considers a brand alliance, the degree of brand fit is assessed in a manner similar to that of assessing product fit. Specifically, because there is generally a lack of an identifiable benchmark in a brand alliance, consumers are likely to search for a higherorder category to establish a link between two brands.

Yet, differences in the nature of products and brands should be highlighted in this discussion. While brands can be considered as categories containing different attributes (Boush and Loken, 1991), these attributes go well beyond the products that compose the brands and the features contained in those products. When the image of a brand is considered, features are expanded to include all perceptions that reflect consumer associations in memory (Keller, 1993). Such associations are likely to be meaning oriented and symbolic rather than strictly functional as are features. Brand fit, therefore, is more likely to be based on an evaluation of image (luxurious, high tech) than attributes (leather interior, fast processor speed).

A brand-oriented application of a theory known as "conceptual consistency" will help to explain how higher-order categorization applies to the evaluation of brand fit. This theory recognizes that attribute comparison alone does not account for the evaluation of fit of two distinct objects as set forth by feature matching as in brand extension research (Murphy and Medin, 1985; Park et al., 1991). Aside from a relationship between object attributes, two objects may "fit" together because they share some concept. "Brand concepts are brand-unique abstract meanings (e.g., high status) that typically originate from a particular configuration of product features (e.g., high price, expensive-looking design, leather interior, etc.) and a firm's efforts to create meanings from these arrangements" (e.g., "the relentless pursuit of perfection" by Lexus; Park et al., 1991, page 186). Notice that the features and the concept given here refer to the same category, luxury cars.

The idea of coherence between two different concepts can be understood based on higher-order categorization. In essence, a concept is a category. Thus, to the extent that a common higher-order concept can be used to group the two lower order concepts, consistency or fit between the two concepts can be established. As a means of illustrating, consider the brands Mercedes Benz and Rolex. On a product feature basis, these brands seem to share little in common, even when a higher-order category is sought. However, both of these brands are associated with a very exclusive high-status concept. While the products made by each brand may not seem complementary, the concepts are consistent resulting in a high level of brand fit.

Any given brand can be associated with multiple concepts. The concepts that are accessed at any particular time will depend upon the relevance or salience of the concept to the context. When one brand is put in the context of another brand, the concept(s) that emerges as relevant will be one (if one exists) that bears some similarity to the concept(s) of the second brand.

\section{HYPOTHESES}

This literature review has given a discussion of higher-order categorization describing the level of abstraction of categories in a taxonomy (Mervis and Rosch, 1981). In considering both the product and brand components of a brand alliance, the level of abstraction of one relative to the other should be considered. Based on the concepts reviewed in the previous section, products by their very nature are more concrete than brands. Products are based on hard, observable features and are thus more readily and consistently defined. It should also be noted that while broad, more abstract product categories do exist (i.e., "consumer electronics"), when a product is shown in an ad, it is easily applied to the more concrete subset (i.e., "CD player" or "alarm clock"). In contrast, because brands include not only associated product features but many other associations relevant to brand image, brands are more abstract in nature than products. It follows then that: 
Hypothesis 1: The evaluation of product fit is based on binding higher-order categories that are concrete while the evaluation of brand fit is based on binding higher-order categories that are abstract.

While Hypothesis 1 is based on a comparison of the level of abstraction of binding higher-order categories between dimensions, this level of abstraction should also be examined within each dimension. The application of hierarchical categorization research, particularly in the field of marketing (Meyers-Levy and Tybout, 1989), demonstrates that a better fit between two objects results when they can be grouped by a higher-order category that is more concrete than abstract. Thus, it is proposed that:

Hypothesis 2: As the level of product fit increases, the level of abstraction of a binding higher-order category decreases, becoming more concrete than abstract.

In the literature review of this paper, it was illustrated that products in a brand alliance are compared based on the features of a higher-order category, rather than the features at the product category level. Product level features are not compared to each other for their similarity (standard feature matching). It is proposed here that as similarity is sought through a higher-order category, lower-order product level features are compared based on their complementarity rather than their similarity. This complementarity is based on a uses orientation. In other words, because the search for a binding higher-order category is likely to be driven by concrete features, the features involved in the higher-order category should reflect how the two products can be used together (i.e., "airline service" and "credit card service" combined together as "services useful for travel").

In contrast, an evaluation of brand categories involves a comparison of the images of each brand and is more conceptual in nature. Brand image can include all pieces of information associated with a brand, including attributes, characteristics, benefits, and attitudes. In this manner, it is proposed here that in evaluating brand fit, any binding higher-order category should reflect a similarity or consistency of the images of the two brands.

Additionally, based on the discussion of higher-order categorization in the previous section, it should be apparent that the weaker the level of fit on either the product dimension or the brand dimension, the more abstract any possible binding higher-order category will be. Thus, when assessing a brand alliance with a strong degree of product fit, the features that drive a search for a higher-order product category will be readily apparent. Additionally, a common usage based on the complementarity of product features should be more readily assessable than in a brand alliance with a low level of product fit. In the same manner, the evaluation of a brand alliance with strong brand fit should more readily generate a common concept or image. Thus,

Hypothesis 3A: Product fit is evaluated based more on the uses of each product, relative to brand fit which is evaluated based on the images of the brands.

Hypothesis 3B: Product combinations with high levels of fit will result in fit evaluations that are based more on the uses of each product than product combinations with low levels of fit.

Hypothesis 3C: Brand combinations with high levels of fit will result in fit evaluations that are based more on the images of each brand than brand combinations with low levels of fit.

\section{METHODS}

Stimuli for this study were created by pretesting various combinations of brands and products for levels of brand fit and product fit. A three-item semantic differential scale for assessing brand and product fit was used (is/is not consistent, is/is not complementary, is/is not expected; see Simonin and Ruth, 1998). Based on the pretesting, five product combinations with low levels of fit and five product combinations with high levels of fit were chosen. Five brand combinations with high and low levels of fit were also chosen.

120 students at a major southwestern university participated in this study. While the disadvantages of using student participants with respect to external validity can be argued, student participants provide the advantage of a homogeneous sample. Thus, the error variance due to such factors as age, education, work experience, and income 
is reduced (Calder, Phillips and Tybout, 1981). Such internal validity is further enhanced as all pretests for developing the brand alliances used in this study drew a participant sample from the same pool during the same time period.

Each participant was given a survey. On the front page were detailed instructions including a description of advertising involving the strategy of brand alliance and an example of how brand/product pairs vary as to their level of fit. Each survey consisted of either five brand pairs or five product pairs. A brand/product pair was listed at the top of each page of the survey packet with instructions for respondents to consider that brand/product pair for inclusion in a brand alliance. Ultimately, each pair was rated by approximately 30 respondents.

Five questions designed to assess a person's thoughts while evaluating the fit of each pair of products were then asked. These questions included three quantitative measures based on single item seven-point semantic differential scales (numbers 1, 4, and 5) and two qualitative open-ended measures (numbers 2 and 3). The form for brand pairs was essentially identical, with only minor modifications to accommodate the difference between brand and product. Question 1 had two purposes: 1) to assess the level of brand/product fit in a very general manner, and 2) to provide a foundation upon which to base the following questions. Question 2 provided an in-depth measure of the reasons for the respondents' assessments of fit.

Question 3 was designed to measure the ability to create a higher order category into which both components would fit. Respondents were asked to give this higher order category in the form of a headline for an ad that would provide a logical central theme for the two components. Questions 4 and 5 were designed to provide quantitative measures for the level of concreteness of the higher order category given by the respondent in question 3 .

\section{RESULTS}

Two single-item seven-point semantic differential scales were used to code the degree to which the responses to Question 2 mentioned the uses (USES) and the overall images (IMAGES) of the product/brand pair (not at all/a great deal) in order to test Hypotheses 3a, 3b, and 3c. Two additional single-item seven-point semantic differential scales were used to code the degree to which the headlines given in response to Question 3 provided a logical (LOGIC) binding higher-order category (not at all/a great deal) as well as the degree to which the headlines were unique (UNIQUE) to the particular product/brand pair (not at all unique/very unique). These two variables provide separate measures for the level of abstraction of the given higher-order categories.

The fit manipulation check provided by Question 1 supported the pre-tested fit conditions. Product combinations in the high fit condition rated higher than those in the low fit condition (5.93 vs 3.00; $<<.0001)$. Similarly, brand combinations in the high fit condition rated higher than those in the low fit condition (5.07 vs $2.81, \mathrm{p}<.0001)$.

Hypothesis 1 identifies the involvement of higher-order categorization in the evaluation of brand alliance fit and proposes a comparison of the level of abstraction between dimensions. Hypothesis 1 was tested by assessing the level of abstraction of higher-order categories used to group product and brand combinations through the LOGIC and UNIQUE variables.

An examination of these two measures gives evidence of a distinction between brand and product fit based on the level of abstraction (see Table 1). The headlines given by participants identifying higher-order categories were judged to be a more logical grouping mechanism (LOGIC) for product combinations than for brand combinations (F $=6.005, \mathrm{p}<.02$ ). In addition, levels of UNIQUE were higher for product combinations than for brand combinations $(\mathrm{F}=14.47, \mathrm{p}<.0001)$. Thus, support is achieved for Hypothesis 1 . 
Table 1. Means For Level Of Abstraction Measures (Between)

\begin{tabular}{l|cc}
\hline & Product Fit & Brand Fit \\
Mean (SD) & Mean (SD) \\
\hline Logic & $4.185(1.92)$ & $3.64(1.70)$ \\
\hline Unique & $5.41(1.63)$ & $3.79(2.57)$ \\
\hline
\end{tabular}

As a means of illustrating that product pairs in a brand alliance are grouped by higher-order categories that are more concrete than those used to combine brand pairs, consider the following examples of ad headlines. In response to the product combination of a microwave oven and food containers, one participant suggested the headline "The Cook and Store Combo". This headline reflects the concrete nature of product fit based on the relevance to two identifiable, concrete product categories. In contrast, one participant suggested this headline for the brand combination of Levi's and Airwalk: "It's all about comfort." This headline is much more conceptual and, therefore, abstract in nature as is evident by the fact that it could be applied to any number of brands.

Hypothesis 2 addresses the roles that categorization processes play within both product fit and brand fit. The level of product fit should have a negative relation with level of abstraction for categorization. In other words, a high level of product fit should result in a more concrete higher-order category than a low level of fit. However, due to the abstract nature of brand images and the assessment of brand fit, the categorization process should not result in a significant difference in the level of abstraction between strong brand fit and weak brand fit.

The variables LOGIC and UNIQUE were again used for assessing the level of abstraction of higher-order categories given by participants. Partial support is found for Hypothesis 2 based on ANOVA of the variable LOGIC $(\mathrm{F}=$ $272.09, \mathrm{p}<.0000)$. While the difference between high product fit $($ Mean $=5.42)$ and low product fit $($ Mean $=2.86)$ is strong here, the difference between the fit levels based on UNIQUE is not statistically significant $(\mathrm{F}=2.271, \mathrm{p}=$ .1333).

The final set of hypotheses deals with the characteristics that are involved in assessing the fit levels of product and brand pairs. Hypotheses 3A-C not only identify distinctions between the assessment of both types of fit, but distinctions between high and low levels within each type of fit. The results from analyzing the fit protocols show strong support for each of these hypotheses. On a seven-point scale, participant protocols reflected higher levels of USES in evaluations of product fit than in evaluations of brand fit $(\mathrm{F}=99.13, \mathrm{p}<.0001)$. In a similar manner, protocols reflected a higher level of IMAGES in evaluations of brand fit than in evaluations of product fit $(\mathrm{F}=$ 157.75, $\mathrm{p}<.0001$ ). Thus, support is provided for Hypothesis 3A (see Table 2).

Table 2. Fit evaluation scores for product and brand fit (between)

\begin{tabular}{l|cc}
\hline & Product Fit & Brand Fit \\
Mean (SD) & Mean (SD) \\
\hline Uses & $4.09(2.17)$ & $2.14(1.65)$ \\
\hline Images & $1.46(1.06)$ & $3.2(2.06)$ \\
\hline
\end{tabular}

This dynamic is also apparent in the following participants' responses:

Product combination of microwave oven and food containers - "Microwave oven and food storage containers meet numerous dependencies. Containers must hold left over food which is then microwaved. These would be good combinations. People buying microwave ovens may be starting their 'first' kitchen and they would need these containers."

Protocol for brand combination of Levi's and Airwalk - "Levi's and Airwalk have casual lifestyle images. Airwalk appeals to a younger audience, so I think it would be good for Levi's to piggy-back into that market on Airwalk's appeal." 
The product protocol given here is a perfect example of how the fit between the two products in question is explained in terms of the uses for each product and how the two can be used together. On the other hand, the brand protocol does not mention uses at all. It focuses on the casual nature of both brands and their appeal to a youth market reflecting a clear image orientation.

A specific examination of the levels of product fit and brand fit provides support for Hypotheses 3B and 3C (see Table 3). Product combinations in a high fit condition are more readily processed based on uses of the products than

Table 3. Fit evaluation scores for product and brand fit (within)

\begin{tabular}{l|cc}
\hline & $\begin{array}{c}\text { Product Pairs } \\
\text { Uses Scores } \\
\text { Mean (SD) }\end{array}$ & $\begin{array}{c}\text { Brand Pairs } \\
\text { Image Scores } \\
\text { Mean (SD) }\end{array}$ \\
\hline High Fit & $4.93(1.84)$ & $3.61(2.2)$ \\
\hline Low Fit & $3.23(2.11)$ & $2.78(1.82)$ \\
\hline
\end{tabular}

are product combinations in a low fit condition $(\mathrm{F}=63.54, \mathrm{p}<.0001)$. The examination of brand fit levels similarly reveals that evaluations of fit for brand combinations in the high fit condition are based on the images of each brand more than for brand combinations in the low fit condition $(\mathrm{F}=6.54, \mathrm{p}<.01)$. This support not only gives evidence of the distinction in the nature of the dimensions of brand fit and product fit, but gives evidence that as the level of fit on either dimension decreases, it becomes more difficult to find a higher order category to group the components under consideration.

\section{DISCUSSION}

It was stated previously that the purposes of this paper were to establish the process involved in making evaluations of product fit and brand fit, and to provide further evidence of the distinction between these two dimensions of brand alliance fit. Support of the three hypotheses in this paper accomplishes both of these purposes.

One of the tasks that participants completed as part of the experiment was to create a headline that would provide a binding theme or category for the brand/product pair featured in the ad. The fact that participants were able to do this provides some evidence that a process of searching for a higher-order category is conducted when making evaluations of fit. More importantly, it was proposed that due to the nature of products and brands as subordinate categories, higher-order categories grouping product pairs would be more concrete than higher-order categories grouping brand pairs. Confirmation of Hypothesis 1 provides more convincing evidence of the involvement of categorization in brand fit evaluations.

Providing further evidence that categorization occurs when processing brand alliances, results supporting Hypothesis 2 show that higher-order categories are more concrete when product fit is high relative to when product fit is low. This confirms what prior categorization research has shown - when two categories have a high level of fit at a subordinate level, a binding higher-order category can be found within a level of the taxonomy that is closer and at a lower level of abstraction than when two subordinate categories have a low level of fit.

As final support for the existence of higher-order categorization in brand and product fit evaluations, consider that previous research has shown that establishing a higher-order category for closely tied items requires less cognitive effort and is therefore easier than conducting this same task for loosely tied items (Mandler, 1982; Mervis and Rosch, 1981; Myers-Levy and Tybout, 1989). That this same dynamic occurs in the processing of brand and product fit is evident in support for Hypotheses 3A, 3B, and 3C. This current research proposed that product pairs would be evaluated based on complementarity of uses and that brand fit would be evaluated based on complementarity of images. Not only was this confirmed, but it was shown that this occurred significantly more at high levels of product and brand fit than at lower levels. This is indicating that when fit is low, more cognitive effort is required, the task is more difficult, and the outcome is, therefore, more vague. 
While it is clear from these results that consumers organize brand alliance information based on a hierarchy of categories, it should also be noted that these same results supporting all three hypotheses also provide confirmatory evidence as to the existence and distinction between two dimensions of brand alliance fit. This is significant. While various studies have addressed the issue of fit or complementarity of brand alliance components, few have identified and established that brand alliance fit consists of multiple fit dimensions (Hao, 2015; Simonin and Ruth, 1998).

\section{FUTURE RESEARCH AND LIMITATIONS}

By establishing that product and brand fit are ascertained through a process of hierarchical categorization, an entire field of research can be applied to the study of how consumers process brand alliances. The studies that have established this area of research have examined variables that have an effect on attitudes toward the alliance, attitudes toward each component brand, beliefs about the brands involved, and how accessible those beliefs are (Bendik, 2015; Hao, 2015; Rao et al., 1999; Samu et al., 1999; Simonin and Ruth, 1998). However, there remains a great deal to be learned about how brand alliance information is processed and what factors affect both cognitive and evaluative outcomes. Applications of categorization theories open new avenues in the study of this area of consumer research.

One such example is applying Mandler's hypothesis of schema incongruity (Mandler, 1982). This theory describes how varying degrees of schema congruity and incongruity can result in differing outcomes on affective responses to the stimuli. Specifically, Mandler establishes that items of moderate incongruity result in more positive affective responses than items of extreme congruity or extreme incongruity due to the degree of elaboration required to process the varying levels of incongruity. This theory has been applied by operationalizing schema incongruity based on a hierarchical category structure in the context of new product introduction (Meyers-Levy and Tybout, 1989). Based on this precedent, Mandler's hypothesis could be applied in the context of brand alliances.

Additionally, there are implications of categorization processes on cognitive outcomes of brand alliance processing. Most apparent is the fact that varying degrees of category abstraction or schema congruity require different levels of elaboration. This should have direct effects on memory storage, retention, and recall. It has been shown that depending on the type of elaboration, a comparison of content can lead to an evaluation based on both distinctive (item-specific elaboration) and similar (relational elaboration) characteristics (Meyers-Levy, 1991). Thus, both low and high levels of elaboration can exhibit beneficial effects on the cognitive outcomes of category specific processing.

While this current research makes a significant contribution to the understanding and application of brand alliances, this study is not without limitations. The suggestions given in this section for future research provide some context for limitations. Certainly, an understanding of the cognitive processes involved in evaluations of brand alliances, particularly with respect to hierarchical categorization, are to some extent limited without empirical studies of brand alliances investigating elaboration and schema incongruity. Furthermore, the methodology employed for this research relied on convenience sampling by employing student participants. While it has been noted that such sampling has advantages in establishing internal validity, it has limitations with respect to external validity. In order to establish stronger generalizability of these current findings to a broader population, future research should involve sample elements with greater variability on such factors as income, age, experience, need, ability to purchase, and perceptions of utility in relation to brand alliance evaluations. Non-student sample elements will also alleviate any possible effects of participant willingness and authority on evaluative outcomes.

\section{AUTHOR BIOGRAPHY}

Dr. Andrew Norman is an Associate Professor of Marketing at Drake University where he has served since 2003. With degrees from the University of Arizona ( $\mathrm{PhD}$ in Marketing), Brigham Young University (MBA), and Arizona State University (BA in Communication), Dr. Norman's work in marketing alliances and entertainment consumption has been published in top marketing journals, including the Journal of Consumer Research and the Journal of Retailing. Dr. Norman also contributes research and content to Kotler and Armstrong's Principles of Marketing and Armstrong and Kotler's Marketing: An Introduction. His experience also extends to executive education courses for major corporations. 


\section{REFERENCES}

Aaker, D.A. \& Keller, K.L. (1990). Consumer evaluations of brand extensions. Journal of Marketing, 54(1), 27-41.

Arnett, D.B., Laverie, D.A. \& Wilcox J.B. (2010). A longitudinal examination of the effects of retailer-manufacturer brand alliances: The role of perceived fit. Journal of Marketing Management, 26(1-2), 5-27.

Bendik, S. (2015). The multiple roles of fit between brand alliance partners in alliance attitude formation. Marketing Letters, 26(4), 619-629.

Bhat, S. \& Reddy, S. (2001). The impact of parent brand attribute associations and affect on brand extension evaluation. Journal of Business Research, 53(3), 111-122.

Boush, D.M. \& Loken, B. (1991). A process-tracing study of brand extension evaluation. Journal of Marketing Research, 28(1), 16-28.

Broniarczyk, S.M. \& Alba, J.W. (1994). The importance of the brand in brand extension. Journal of Marketing Research, 31(2), 214-228.

Calder, B.J., Phillips, L.W., \& Tybout, A.M. (1981). Designing research for application. Journal of Consumer Research, 8(2), 197-207.

Hao, W. (2015). Reexamination of brand alliance evaluation model: The effects of the overall fit between partner brands on consumer brand alliance evaluation. Academy of Marketing Studies Journal, 19(1), 11-23.

James, D. (2005). Guilty through association: brand association transfer to brand alliances. Journal of Consumer Marketing, 22(1), 14-24.

Levin, I.P., \& Levin, A.M. (2000). Modeling the role of brand alliances in the assimilation of product evaluations. Journal of Consumer Psychology, 9(1), 43-52.

Keller, K.L. (1993). Conceptualizing, measuring, and managing customer-based brand equity. Journal of Marketing, 57(1), 1-22.

Kreuzbauer, R. \& Malter, A.J. (2005). Embodied cognition and new product design: Changing product form to influence brand categorization. Journal of Product Innovation Management, 22(2), 165-176.

Mandler, G. (1982). The structure of value: accounting for taste. In: Affect and Cognition: The 17th Annual Carnegie Symposium, (ed. by M.S. Clark \& S. T. Fiske, pp. 3-36. Lawrence Erlbaum Associates Hillsdale, NJ, USA.

Mervis, C.B. \& Rosch, E. (1981). Categorization of natural objects. Annual Review of Psychology, 32, 89-115.

Meyers-Levy, J. \& Tybout, A.M. (1989). Schema congruity as a basis for product evaluation, Journal of Consumer Research, 16(1), 39-54.

Meyers-Levy, J. (1991). Elaborating on elaboration: the distinction between relational and item-specific elaboration, Journal of Consumer Research, 18(3), 358-367.

Murphy, G.L. \& Medin, D.L. (1985). The role of theories in conceptual coherence. Psychological Review, 92, 289-316.

Park, C.W., Milberg, S., \& Lawson, R. (1991). Evaluation of brand extensions: the role of product feature similarity and brand concept consistency. Journal of Consumer Research, 18(2), 185-193.

Rao, A.R., Qu L. \& Ruekert, R.W. (1999). Signaling unobservable product quality through a brand ally. Journal of Marketing Research, 36(2), 258-268.

Rao, A.R. \& Ruekert, R.W. (1994). Brand alliances as signals of product quality. Sloan Management Review, $36,87-97$.

Rosch, E. (1978). Principles of categorization. In: Cognition and Categorization (ed. by E. Rosch \& B.B. Lloyd), pp. 27-48. Eribaum, Hillsdale, NJ, USA.

Samu S.H., Krishnan, S., \& Smith, R.E. (1999). Using advertising alliances for new product introduction: interactions between product complementarity and promotional strategies. Journal of Marketing, 63(1), 57-74.

Simonin, B.L. \& Ruth, J.A. (1998). Is a company known by the company it keeps? Assessing the spillover effects of brand alliances on consumer brand attitudes. Journal of Marketing Research, 35(1), 30-42.

Smith, E.E., Shoben, E.J. \& Rips, L.J. (1974). Structure and process in semantic memory: A featural model for semantic decisions. Psychological Review, 81, 214-41.

Smith, E.E. \& Medin, D.L. (1981). Categories and Concepts, Harvard University Press, Cambridge, MA, USA.

Sujan, M. (1985). Consumer knowledge: Effects on evaluation strategies mediating consumer judgments. Journal of Consumer Research, 12(1), 31-46. 